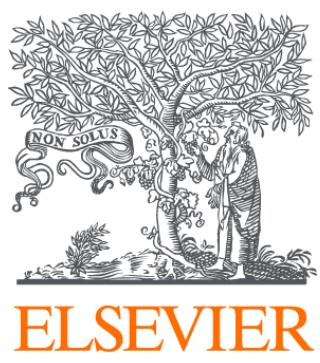

Since January 2020 Elsevier has created a COVID-19 resource centre with free information in English and Mandarin on the novel coronavirus COVID-

19. The COVID-19 resource centre is hosted on Elsevier Connect, the company's public news and information website.

Elsevier hereby grants permission to make all its COVID-19-related research that is available on the COVID-19 resource centre - including this research content - immediately available in PubMed Central and other publicly funded repositories, such as the WHO COVID database with rights for unrestricted research re-use and analyses in any form or by any means with acknowledgement of the original source. These permissions are granted for free by Elsevier for as long as the COVID-19 resource centre remains active. 


\title{
Impact of the COVID-19 pandemic on the surveillance of antimicrobial resistance
}

 \\ ${ }^{a}$ Antimicrobial Resistance Research Center, National Institute of Infectious Diseases, Tokyo, Japan \\ ${ }^{b}$ Department of Bacteriology II, National Institute of Infectious Diseases, Tokyo, Japan
}

\section{A R T I C L E I N F O}

\section{Article history:}

Received 30 April 2021

Accepted 16 September 2021

Available online 22 September

2021

\section{Keywords:}

COVID-19

Antimicrobial resistance

Surveillance

WHO-GLASS

\section{$S U M M A R Y$}

Background: The impact of the coronavirus disease (COVID-19) pandemic on antimicrobial resistance (AMR) is a major concern.

Aim: To compare the number of patients and isolation rate of antimicrobial-resistant bacteria before and after the beginning of the COVID-19 pandemic using the comprehensive national surveillance data.

Methods: We utilized comprehensive surveillance data, collected in the Japan Nosocomial Infections Surveillance programme, which included a total of 16.7 million samples of 5.9 million tested patients from $>1300$ hospitals. We compared the number of patients and isolation rate of five bacteria between 2019 and 2020, including antimicrobial-susceptible and -resistant bacteria of Staphylococcus aureus, Streptococcus pneumoniae, Escherichia coli, Klebsiella pneumoniae and Pseudomonas aeruginosa.

Findings: The number of patients and isolation rate of $S$. aureus and meticillin-resistant $S$. aureus decreased slightly; those of $S$. pneumoniae and penicillin-resistant S. pneumoniae decreased by $60 \%$; and those of third-generation cephalosporin-resistant $K$. pneumoniae increased. The isolation rate of the remaining bacteria apparently increased, although the number of patients decreased. This was due to a substantial decrease in the total number of tested patients (the denominator of the isolation rate), which was larger than that of the number of patients (the numerator of the isolation rate). Consistent results were obtained when the same data were re-aggregated using the procedure of the World Health Organization Global Antimicrobial Resistance Surveillance System, demonstrating the general importance of this problem.

Conclusion: Surveillance data during the COVID-19 pandemic must be carefully interpreted based on examination of the numerator, denominator and background factors that affect the denominator.

(C) 2021 The Author(s). Published by Elsevier Ltd on behalf of The Healthcare Infection Society. This is an open access article under the CC BY-NC-ND license (http://creativecommons.org/licenses/by-nc-nd/4.0/).

\footnotetext{
* Corresponding author. Address: Antimicrobial Resistance Research Center, National Institute of Infectious Diseases, Tokyo, Japan.

** Corresponding author. Address: 4-2-1 Aoba-cho, Higashimurayama, Tokyo, 189-0002, Japan. Tel.: +81 (0)3 52851111.

*** Corresponding author. Address: National Institute of Infectious Diseases: Kokuritsu Kansensho Kenkyujo, Japan. Tel.: +81 (0)3 52851111.

E-mail addresses: akihira@nih.go.jp (A. Hirabayashi), kajihara@nih.go.jp (T. Kajihara), k-yahara@nih.go.jp (K. Yahara).
} 


\section{Introduction}

The coronavirus disease (COVID-19) pandemic has forced major changes to occur in the medical system, including securing hospital beds, providing ventilators, and strengthening infection control measures. To date, the impact of the COVID-19 pandemic on antimicrobial resistance (AMR) has been discussed in several reports [1-5]; topics include a lack of personal protective equipment, increased need for medical care, lack of medical staff, decrease in screening cultures of resistant microorganisms, decrease in laboratory capacity on AMR to focus on COVID-19 diagnosis, and longer duration of antimicrobial therapy due to long-term respiratory management. These factors might have a devastating impact on antimicrobial stewardship and infection control, which could lead to outbreaks of antimicrobial-resistant bacteria (AMRB) in hospitals.

Regarding the isolation rate of AMRB during the COVID-19 pandemic, a report from Singapore [6] indicates that the rates of meticillin-resistant Staphylococcus aureus (MRSA) acquisition in hospital and central-line-associated-bloodstream infections declined significantly owing to aggressive infection prevention control measures to prevent healthcare-associated transmission of COVID-19. In contrast, there are several reports of increased detection rates of multi-drug-resistant Enterobacterales, especially Klebsiella pneumoniae, which suggest increased use of antimicrobials for pneumonia cases during the COVID-19 pandemic $[7,8]$.

However, the majority of previous reports are small and from a single institution. To determine whether the increase or decrease in the isolation rate of AMRB reported in a single medical institution is a general phenomenon in that region and country, it is necessary to analyse large-scale surveillance data. Thus, we utilized comprehensive surveillance data collected in a national AMR surveillance programme, the Japan Nosocomial Infections Surveillance (JANIS), which has been collecting data on all bacteria isolated from all sample types of both symptomatic and asymptomatic patients from clinical laboratories of the participating hospitals since 2000 [9]. As of January 2020, there are 2223 participating hospitals, covering more than $25 \%$ of all hospitals in Japan. The data included more than 8.4 million specimens and over 5.8 million isolates in 2019. In this study, we compared the isolation rates of five types of bacteria, including the antimicrobial-susceptible and -resistant bacteria of S. aureus, Streptococcus pneumoniae, Escherichia coli, K. pneumoniae, and Pseudomonas aeruginosa between 2019 and 2020 (i.e. before and after the beginning of the pandemic), as well as the actual number of patients from which the bacterium was isolated (numerator) and the total number of tested patients (denominator), which can be determined from the comprehensively collected data of all bacteria isolated from the participating hospitals. Furthermore, we conducted a comparison between overall hospitals and those that are mainly responsible for COVID-19 patients as the impact of the COVID-19 pandemic may be stronger in the hospitals responsible for COVID-19 patients.

To reveal the impact of the COVID-19 pandemic, this study aimed to perform a comparative analysis of the actual number of patients and isolation rate of AMRB using the comprehensive national AMR surveillance data before and after the beginning of the COVID-19 pandemic.

\section{Methods}

\section{Data preparation and tabulation}

The number of patients and the isolation rate including infection and colonization for each bacterial species and specific AMRB [9] isolated in approximately 1300 hospitals with $\geq 200$ beds who participated in JANIS, as well as the total number of tested patients in the first, second and third quarters were extracted from the publicly available quarterly reports [10] from 2018 to 2020. The latest report presented was for the third quarter in 2020; therefore, data from the fourth quarter were not extracted in the study. The breakpoints of each antimicrobial were adopted from the Clinical and Laboratory Standards Institute (CLSI) 2012 guidelines.

The number of patients and the isolation rate including infection and colonization of five types of bacteria, including the antimicrobial-susceptible and -resistant bacteria of S. aureus, S. pneumoniae, E. coli, K. pneumoniae, and $P$. aeruginosa, and the total number of tested patients were tabulated by focusing on hospitals responsible for COVID-19 patients with $\geq 200$ beds using inpatient data extracted between January and September in 2019 and 2020 from the JANIS database, which stores both culture-positive and negative test diagnostic results with all antimicrobial susceptibility testing results.

The inpatient data from approximately 16.7 million samples during the period were exported from the database as a text file. This file was sorted according to the facility identification, patient identification, specimen collection date, inpatient or outpatient, and specimen identification, and tabulated using a Java toolkit $[9,11]$. We did not include hospitals with $<200$ beds in this study because they have different characteristics (for example, frequency and timing of specimen collection) compared with those with $\geq 200$ beds, and they are not directly comparable with each other. In addition, tabulation stratified by specimen type was conducted for the number of patients from whom thirdgeneration cephalosporin-resistant E. coli (3GCR-EC) and third-generation cephalosporin-resistant $K$. pneumoniae (3GCR-KP) were detected, in the hospitals responsible for COVID-19 patients, using the Java toolkit.

Furthermore, the inpatient data of hospitals responsible for COVID-19 patients were tabulated according to the procedure defined by the World Health Organization-Global Antimicrobial Resistance Surveillance System (WHO-GLASS) using the Java toolkit [12].

\section{Ethical considerations}

Patient identifiers were de-identified in each hospital before data were submitted to JANIS. The anonymous data stored in the JANIS database were exported and analysed following approval by the Ministry of Health, Labour and Welfare (approval number 0219-7) according to Article 32 of the Statistics Act. 


\section{Results}

\section{Change in the number of patients with any of the five bacterial species during the COVID-19 pandemic}

The number of patients with infection or colonization from any of the five antimicrobial-susceptible bacteria is shown in Figure 1, on the left. The number of patients from whom S. aureus or S. pneumoniae were isolated (red and pink in Figure 1, left) showed a clear decrease from 2019 to 2020; this was more pronounced in the second and third quarters than in the first quarter. Specifically, the number of patients with S. aureus decreased from 85,929 to 73,631 (14.3\% decrease) in the second quarter and from 85,364 to 75,544 (11.5\% decrease) in the third quarter, whereas the number went from 89,704 to 84,465 (5.8\% decrease) in the first quarter. The extent of the decrease in S. pneumoniae in the second and third quarters was much larger than that in S. aureus: the number of patients with S. pneumoniae decreased from 9205 to 3178 (65.5\% decrease) in the second quarter and from 7835 to 3010 (61.6\% decrease) in the third quarter. The number of patients with the remaining three Gram-negative bacterial species ( $E$. coli, K. pneumoniae and $P$. aeruginosa) decreased by $7.3 \%, 7.6 \%$ and $7.2 \%$, in the second quarter, respectively, and decreased by $1.6 \%, 3.2 \%$ and $3.6 \%$, between the third quarter of 2019 and 2020, respectively.

The number of patients with infection or colonization from any of the five AMRB, including MRSA, penicillin-resistant S. pneumoniae (PRSP), 3GCR-EC, 3GCR-KP and carbapenemresistant $P$. aeruginosa (CRP) is shown in Figure 1 , on the right. Among the five AMRB, most notably, the number of patients with MRSA and PRSP showed a marked decrease from 2019 to 2020; the extent of the decrease was much larger in the second and third quarters than in the first quarter, similar to S. aureus and S. pneumoniae; the number of patients with MRSA decreased from 40,758 to 35,264 (13.5\% decrease) in the second quarter and from 40,185 to 35,111 (12.6\% decrease) in the third quarter in 2020 compared with the previous year. The number of patients with PRSP decreased from 3266 to 1318 (59.6\% decrease) in the second quarter and from 2987 to 1311 (56.1\% decrease) in the third quarter of 2020. Among the remaining three Gram-negative AMRB, 3GCR-EC and CRP decreased slightly or showed almost no change in the second and third quarters. The number of patients with 3GCR-EC decreased from 20,506 to 19,892 (3.0\% decrease) in the second quarter and from 20,630 to $20,748(0.57 \%$ increase $)$ in the third quarter; and that with CRP decreased from 4570 to $4109(10.1 \%$ decrease) in the second quarter and from 4859 to $4650(4.3 \%$ decrease) in the third quarter. Notably, the number of patients with 3GCR-KP increased by $>10 \%$ in 2020 , when compared with 2019: it increased from 3142 to 3595 (14.4\% increase) in the second quarter and from 3805 to 4357 (14.5\% increase) in the third quarter.

\section{Comparison of the number of patients and the isolation rate between 2019 and 2020}

The extent and direction of the differences in the isolation rate and number of patients in 2020 compared with 2019 are shown for each of the five bacterial species and specific AMRB (Figure 2 shows the third quarter and Supplementary Figure S2 shows the second quarter), respectively, for all hospitals with $\geq 200$ beds who participated in the surveillance $(N=1326$ in 2019 and $N=1335$ in 2020, blue in Figure 2; $N=1356$ in 2019 and $N=1346$ in 2020, blue in Supplementary Figure S2) and those responsible for COVID-19 patients $(N=404$ in 2019 and $N=393$ in 2020, red in Figure 2 and Supplementary Figure S2). Unexpectedly, except for S. aureus and S. pneumoniae, the isolation rate almost always increased (shifted to the right in Figure 2 and Supplementary Figure S2), although the number of patients decreased. These results were consistent in the second and third quarters. The extent of reduction in the number of patients was always larger in the hospitals responsible for COVID-19 patients than in all hospitals $(P<0.005$, Wilcoxon's rank sum test). However, this was not consistent with the isolation rate.

Exceptionally, 3GC-KP showed an increase in both the number of patients and the isolation rate in 2020 compared with 2019 in both all hospitals and those responsible for COVID19 patients. The number of patients and the isolation rate of 3GC-KP in hospitals responsible for COVID-19 increased by $14.8 \%$ and $34.0 \%$, respectively, and the extent of change was larger than that in all the hospitals.

\section{Difference in the number of patients and percentage of resistance between 3GCR-EC and 3GCR-KP, irrespective of specimen types}

3GCR-KP, which showed an exceptional increase in both the number of patients and isolation rate, was compared with 3GCR-EC (Figure 3) using the data of hospitals responsible for COVID-19 patients in the third quarter in 2019 and 2020 stratified by specimen type. The number of patients with 3GCR-EC (blue bars in Figure 3) decreased in all specimens compared with the previous year, and its percentage in the species (red lines overlaid on the blue bars in Figure 3) remained almost unchanged. In contrast, the number of patients with 3GCR-KP (green bars in Figure 3 ) increased in all specimens, and its percentage in the species also increased from $6.7 \%$ to $7.9 \%$ in respiratory samples, from $7.4 \%$ to $8.3 \%$ in blood samples, from $10.4 \%$ to $12.6 \%$ in urine samples, and from $5.0 \%$ to $7.7 \%$ in stool samples.

\section{Influence of the decrease in the denominator on the isolation rate defined in Japan and the frequency of infections defined in the WHO-GLASS}

The unexpected increase in the isolation rate in 2020 for several bacterial species in spite of the decrease in the number of patients (Figure 2) is explained through examination of the denominator (the total number of tested patients) as follows: when the denominator is compared quarterly between 2019 and 2020 separately for all hospitals and those responsible for COVID-19 patients (Figure 4), the value in the first quarter decreased by $3.8 \%$ and $5.5 \%$, respectively; in the second quarter, it decreased by $14.7 \%$ and $17.6 \%$, and in the third quarter, it decreased by $8.5 \%$ and $14.3 \%$, respectively. In all quarters, the rate of change was greater for hospitals responsible for COVID-19 patients than for all hospitals. For several bacterial species that showed an unexpected increase in the isolation rate in 2020 , the extent of the decrease in the 




Year
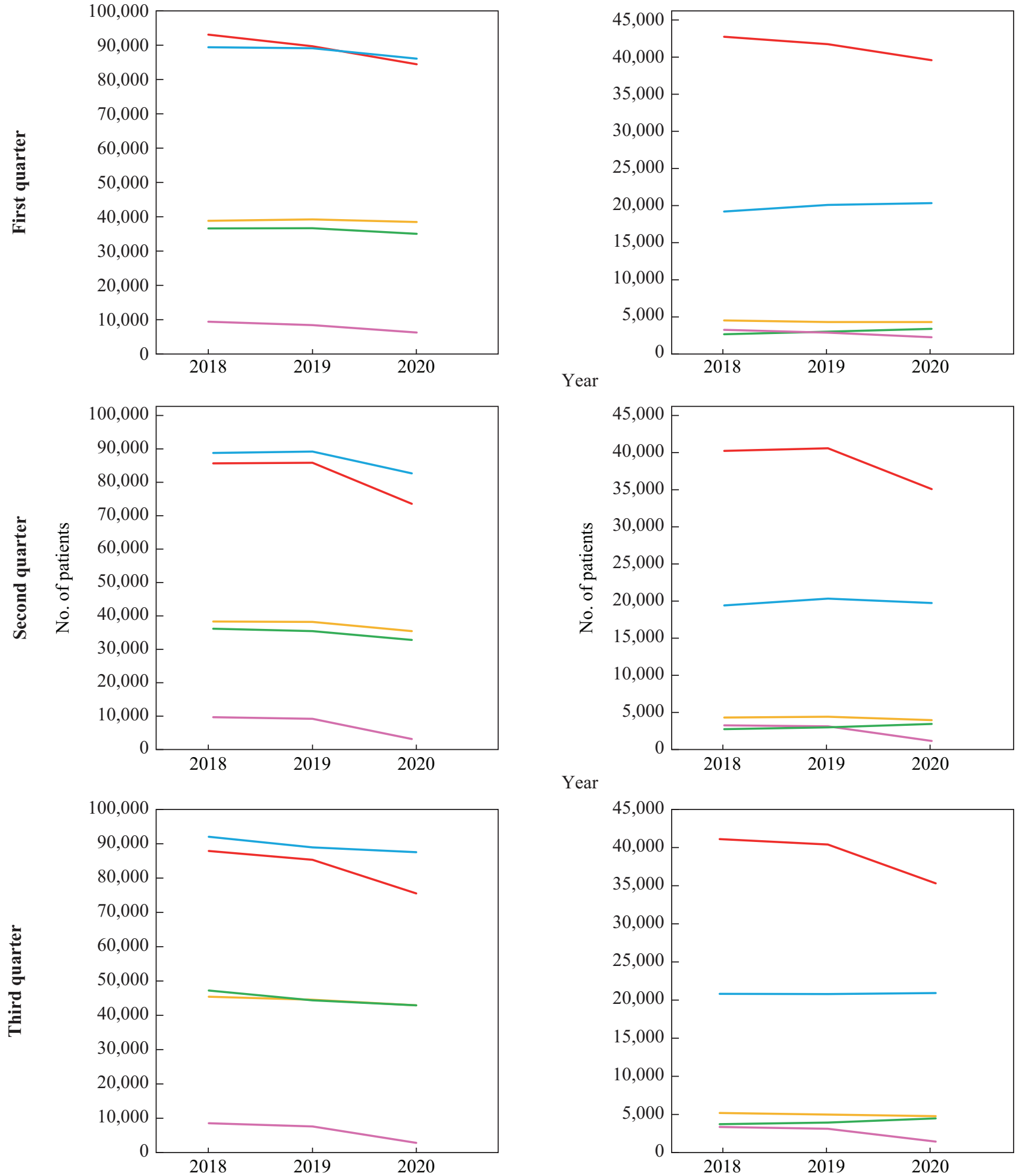

Year



Year

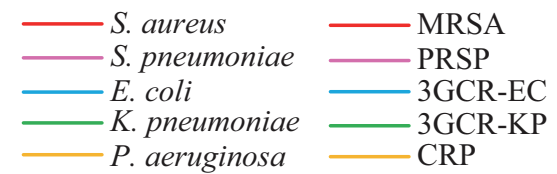

Figure 1. Annual trend of the number of patients from 2018 to 2020 . The three graphs on the left show the numbers of patients from whom each of the five bacterial species were isolated in the first, second and third quarters from 2018 to 2020 . Horizontal axis: years; vertical axis: number of patients. Red bar: Staphylococcus aureus; pink bar: Streptococcus pneumoniae; blue bar: Escherichia coli; green 


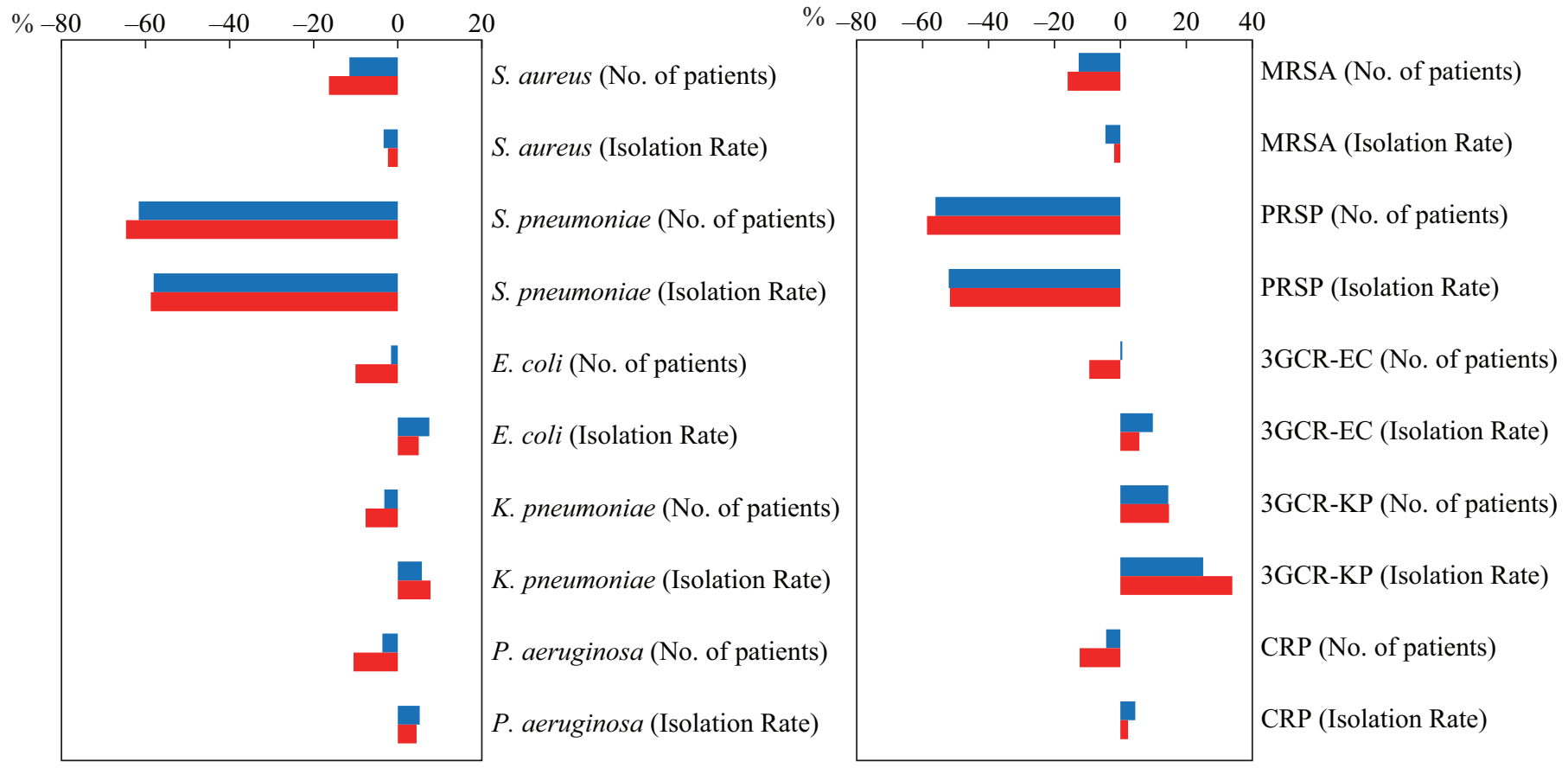

all hospitals $\square$ Hospitals responsible for COVID-19 patients

Figure 2. The difference in the number of patients and isolation rate in the third quarter in 2020 compared with 2019 in all hospitals and hospitals responsible for COVID-19 patients. The graph on the left shows the difference in the number of patients and the isolation rate of the five bacterial species in the third quarter in 2020 compared with 2019, as a percentage. From top to bottom, the differences in the number of patients and isolation rate of Staphylococcus aureus, Streptococcus pneumoniae, Escherichia coli, Klebsiella pneumoniae and Pseudomonas aeruginosa are shown alternately. The graph on the right shows the difference in the number of patients and isolation rate of the five specific antimicrobial-resistant bacteria in the third quarter in 2020 compared with 2019, as a percentage. From top to bottom, the differences in the number of patients and isolation rate of meticillin-resistant $S$. aureus (MRSA), penicillin-resistant S. pneumoniae (PRSP), third-generation cephalosporin-resistant E. coli (3GCR-EC), third-generation cephalosporin-resistant K. pneumoniae (3GCR-KP), and carbapenem-resistant $P$. aeruginosa (CRP) are shown alternately. The blue bar indicates all hospitals $(N=1326$ in $2019, N=1335$ in 2020), whereas the red bar indicates hospitals responsible for COVID-19 patients $(N=404$ in 2019, $N=393$ in 2020).

denominator was larger than that in the numerator (i.e. the number of patients) (Figure 2).

The national AMR surveillance data of $S$. aureus, S. pneumoniae, E. coli, and $K$. pneumoniae in hospitals responsible for COVID-19 in the third quarter were tabulated using the WHO-GLASS method [13], and the extent and direction of the difference in the frequency of infections (corresponding to the isolation rate in JANIS) together with the number of patients in 2020 compared with 2019 is shown for each bacterial species (Figure 5). The frequency of infections defined in the GLASS was calculated per 100,000 tested patients per specimen type. Both the number of patients and frequency of infections markedly decreased for S. pneumoniae, whereas an apparent increase in the frequency of infections despite a decrease in the number of patients was observed for $S$. aureus, $E$. coli, and $K$. pneumoniae isolated from blood, and $K$. pneumoniae isolated from urine. These results were almost consistent with those from JANIS [9], as shown in Figure 2.

\section{Discussion}

We divided the patterns of the results of the extent and direction of the difference in the number of patients and isolation rate into the following three categories: Category 1 includes bacteria whose number of patients and isolation rate are both decreasing; Category 2 includes the bacteria whose number of patients and isolation rate are both increasing; and Category 3 includes the bacteria whose number of patients is decreasing, but the isolation rate is apparently increasing. These trends were more pronounced in the second and third quarters than in the first quarter for all categories, and seemed to occur as the number of COVID-19 patients increased [14].

Category 1 included S. aureus, MRSA, S. pneumoniae and PRSP. In general, the detection rate of MRSA is often monitored as an indicator of hand hygiene [15], but the colonization and style of transmission of meticillin-susceptible S. aureus (MSSA) is similar to that of MRSA [16]. The decrease in S. aureus and

bar: Klebsiella pneumoniae; and yellow bar: Pseudomonas aeruginosa. The three graphs on the right show the numbers of patients from whom each of the five specific antimicrobial-resistant bacteria were isolated in the first, second and third quarters from 2018 to 2020 . Red bar: meticillin-resistant S. aureus (MRSA); pink bar: penicillin-resistant S. pneumoniae (PRSP); blue bar: third-generation cephalosporinresistant E. coli (3GCR-EC); green bar: third-generation cephalosporin-resistant $K$. pneumoniae (3GCR-KP); yellow bar: carbapenemresistant $P$. aeruginosa (CRP). 
Respiratory tract

$$
\% \frac{3 \mathrm{GCR}}{3 \mathrm{GCS}+3 \mathrm{GCR}}
$$

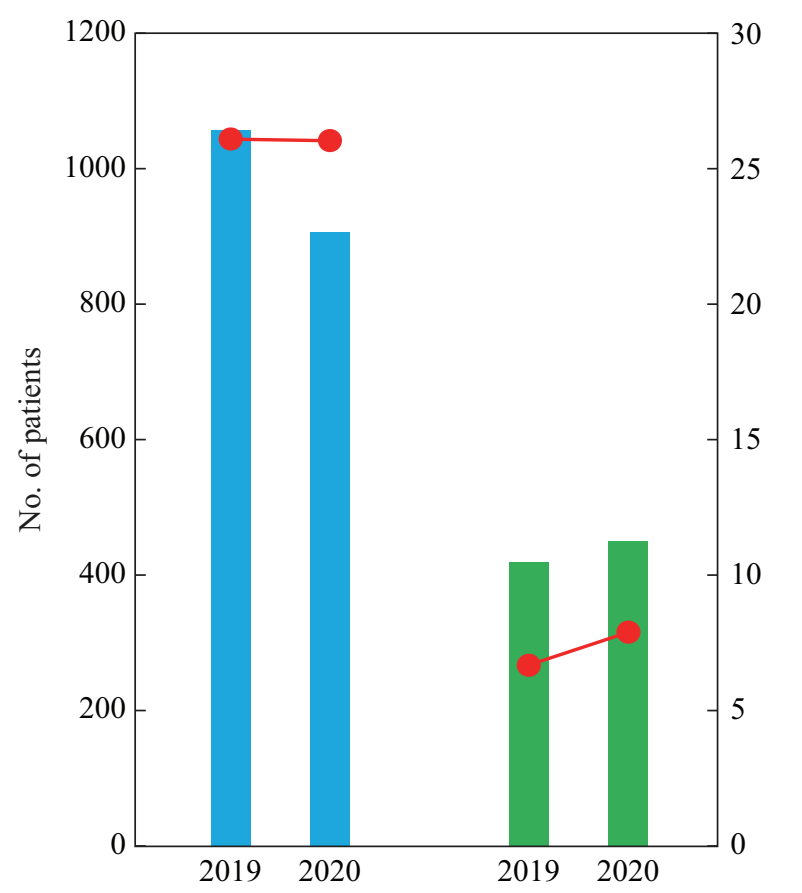

Blood
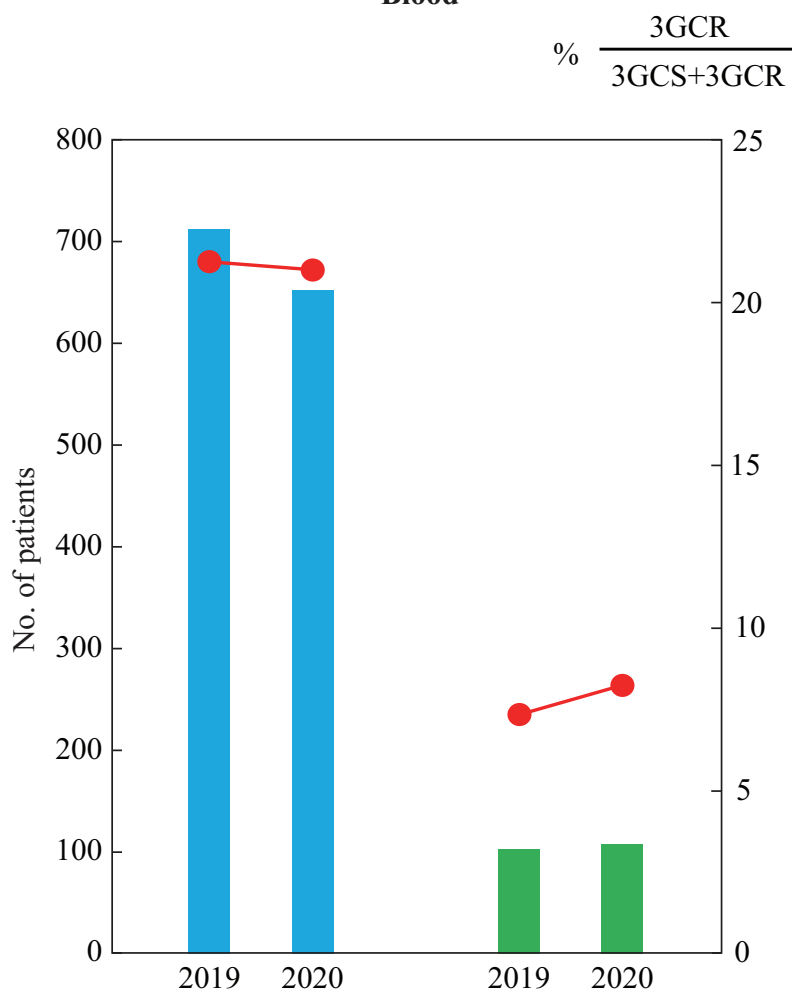

Urine

$$
\% \frac{3 \mathrm{GCR}}{3 \mathrm{GCS}+3 \mathrm{GCR}}
$$



Stool

$\% \frac{3 \mathrm{GCR}}{3 \mathrm{GCS}+3 \mathrm{GCR}}$

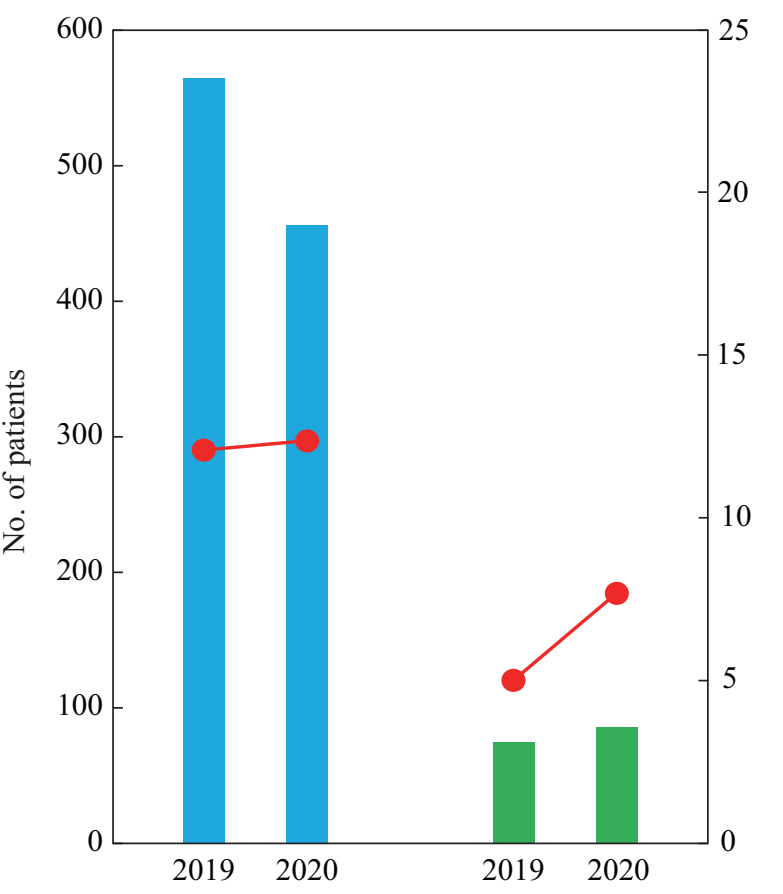

3GCR-KP

Figure 3. The number of patients with third-generation cephalosporin-resistant Escherichia coli (3GCR-EC) and third-generation cephalosporin-resistant Klebsiella pneumoniae (3GCR-KP) and the percentage of patients from whom strains resistant to third-generation cephalosporins were isolated in hospitals responsible for COVID-19 patients stratified by specimen type. The $y$-axis on the left side 




First quarter

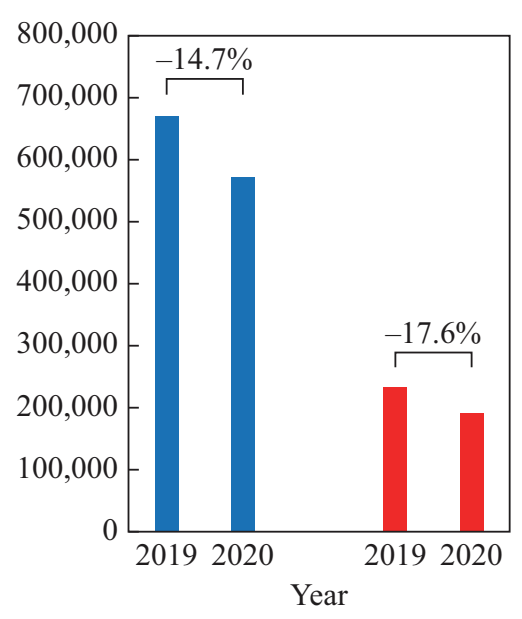

Second quarter

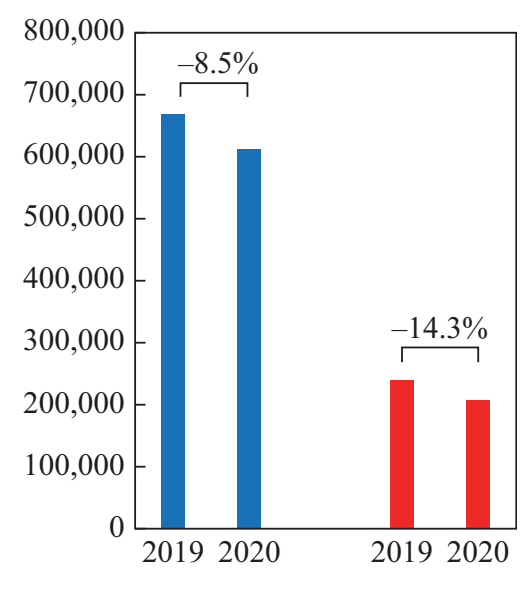

Third quarter

- All hospitals Hospitals responsible for COVID-19 patients

Figure 4. The total number of tested patients in 2019 and 2020. The three graphs show the total number of tested patients on the vertical axis, and the year on the horizontal axis, for each quarter. The numbers in the graphs indicate the percentage of the difference in 2020 compared with 2019. The blue bar indicates all hospitals and the red bar indicates the hospitals responsible for COVID-19 patients.

MRSA may reflect the results of more thorough infection control measures for hand hygiene and other contact prevention measures in hospitals, as the number of patients with COVID-19 increases. To verify this, it will be necessary to examine the correlation between the amount of disinfectant used and the reduction of $S$. aureus or MRSA in further studies. S. pneumoniae and PRSP have decreased markedly in terms of both the number of patients and the isolation rate. In Japan, a state of emergency was declared due to the COVID-19 pandemic for a month from April to May in 2020, which is during the second quarter of 2020, and there were restrictions on unnecessary outings during that time. After that, the Ministry of Health, Labour and Welfare published a 'new way of life', which included maintaining a social distance, wearing of masks, and washing of hands, and this has been practiced widely by citizens to date. As a result, the incidence of infections caused by S. pneumoniae and PRSP has decreased. There is an article on the decline in the incidence of invasive S. pneumoniae infections in children's hospital in the USA during the COVID-19 pandemic; the study also discussed the association of the declining trend with social distancing, wearing of masks and school lockdown [17].

Category 2 includes bacteria whose number of patients and isolation rate both increased: only 3GCR-KP was found in the present study. According to previous reports, the increase in multi-drug-resistant $K$. pneumoniae may be due to the increased use of antimicrobials or nosocomial transmission $[7,8]$. Compared with the actual frequency of bacterial co-infection in COVID-19 patients, frequency of antimicrobial use is higher [18,19]. Several studies have reported that extended-spectrum beta-lactamase (ESBL)-producing $K$. pneumoniae is more likely to cause nosocomial transmission than ESBL-producing E. coli [20-23]. Moreover, in these ESBL- producing strains, biofilm production, which is one of the most important factors of nosocomial dissemination, is linked to certain virulence factors in $E$. coli, but is common in K. pneumoniae [24]. Continuous monitoring of surveillance data and analyses of bacterial side factors of 3GCR-KP will be necessary.

The unexpectedly found Category 3 included the following bacterial species and specific AMRB: E. coli, K. pneumoniae, $P$. aeruginosa, 3GCR-EC, and CRP (Figure 1). Although the number of patients with each bacterial species (i.e. the numerator) decreased, the extent of the decrease in the total number of tested patients (i.e. the denominator) was larger, resulting in an apparent increase in the isolation rate defined in Japan and the frequency of infections defined in the WHOGLASS. One of the reasons for the decrease in the total number of tested patients would be that the increase in the number of newly polymerase chain reaction-positive COVID-19 patients and hospitalized COVID-19 patients in the second and third quarters caused a decrease in the number of hospital admissions for other diseases and increased the testing threshold due to the risk of transmission of COVID-19. The decrease in the total number of tested patients was much larger in hospitals for COVID-19 patients than in all hospitals (Figure 4); this is consistent with the preceding consideration.

The results of Category 3 suggest that in AMR surveillance, a simple comparison of the isolation rate or frequency of infections over time is possible if the total number of tested patients (denominator) does not fluctuate significantly among hospitals between the compared time points. Otherwise, as in the case of the COVID-19 pandemic in 2020, it is necessary to interpret the isolation rate or frequency of infections based on consideration of the background that led to a substantial increase or decrease in the denominator.

shows the number of patients from whom 3GCR-EC and 3GCR-KP were obtained in the third quarter in hospitals responsible for COVID-19. The $y$-axis on the right side shows the percentage of patients from whom strains resistant to third-generation cephalosporins were isolated: $3 G C R /(3 G C S+3 G C R)$ where 3GCS represents 'third-generation cephalosporin-susceptible'. The number of patients with $3 G C R$ EC is shown in blue and that of patients with 3GCR-KP is shown in green. The percentage is shown in a red plot. 


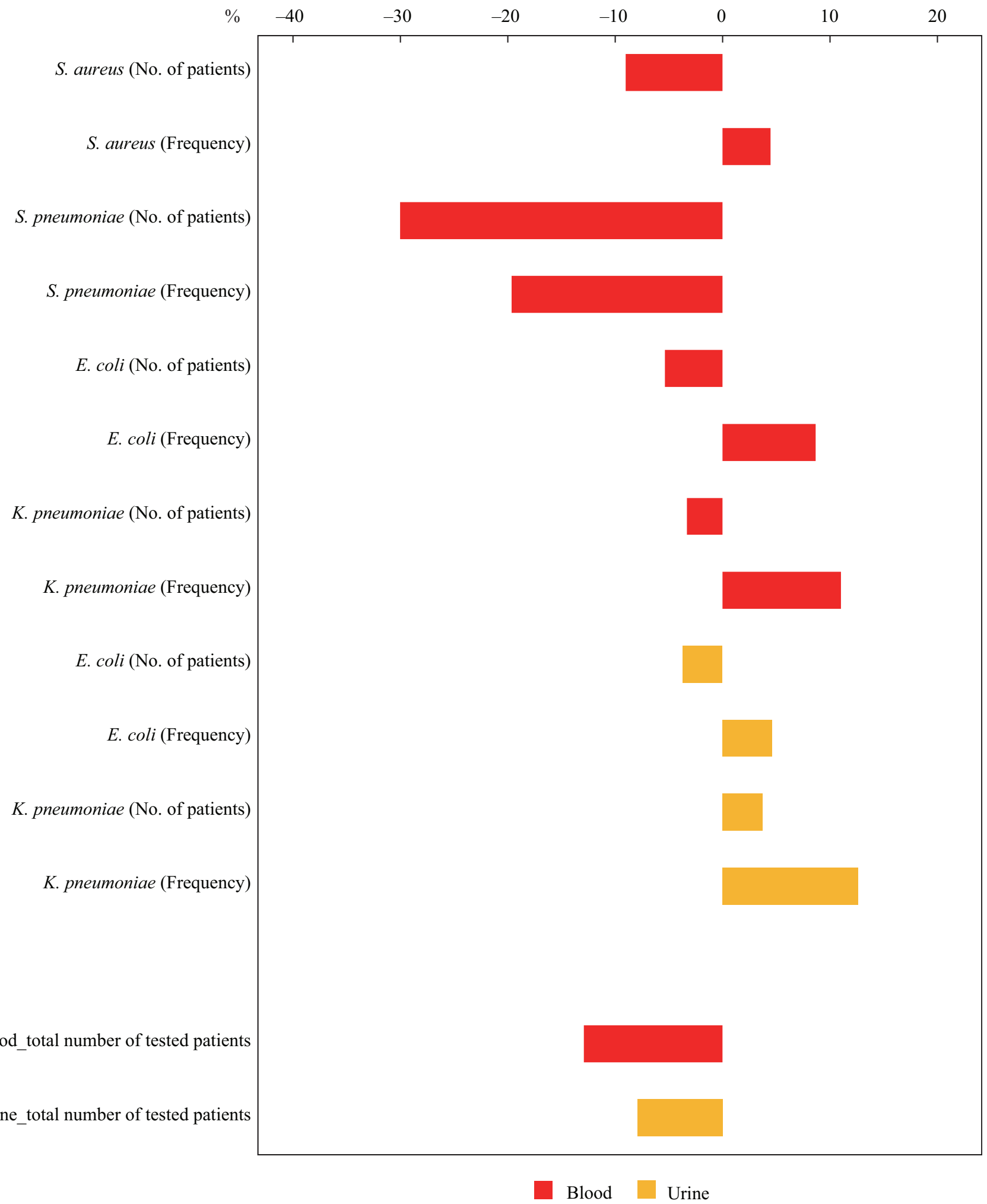

Figure 5. Differences in the number of patients and frequency of infections defined in the World Health Organization - Global Antimicrobial Resistance Surveillance System (WHO-GLASS) in the third quarter in 2020 compared with 2019 in hospitals responsible for COVID-19 patients. The graph shows the differences in the number of patients and the frequency of infections of each bacterial species in the third quarter in 2020 compared with 2019, as a percentage. From top to bottom, the differences in the number of patients and frequency of infections of Staphylococcus aureus, Streptococcus pneumoniae, Escherichia coli and Klebsiella pneumoniae are shown alternately. Red bar: bacteria isolated from blood; yellow bar: bacteria isolated from urine. The frequency of infections indicates the number per 100,000 tested patients according to the GLASS method. 
Regarding the background situations and issues that may affect the denominator in AMR surveillance, a previous study showed the several factors that affect the denominator: number of beds, number of admissions, number of hospitalization days, and number of tested samples [25]. These background factors can change considerably with situations such as the participation of new hospitals with different characteristics and frequencies of testing, a major outbreak of AMRB, and an infectious disease pandemic, such as the COVID-19 pandemic, as we demonstrated in this study. Other previous studies have reported an apparent increase or decrease in the incidence or mortality rate, as a result of changes in background factors such as introduction of new screening protocols, which have led to an increase in the number of diagnosed cases [26,27]. A previous surveillance study reported an apparent rise in a pathogen incidence due to an increase in the number of tested stool samples from the elderly [28]. In the present study, we quantitatively demonstrated that the same problem could occur in both isolation rate defined in Japan and the frequency of infections defined in WHO-GLASS (Supplementary material) using the total number of tested patients. Indeed, tabulation of the national surveillance data in Japan using the GLASS method also revealed that the frequency of infections apparently increased in spite of the decrease in the number of patients with the bacteria (Figure 5), similar to the isolation rate defined in JANIS (Figure 2). Therefore, the importance of denominator in AMR surveillance is a worldwide problem, rather than one confined to Japan. The main limitation of this study is because JANIS has been collecting data on all bacteria isolated from all sample types without making a distinction between infection and colonization, the impact of the COVID-19 pandemic on infections caused by AMRB has not been assessed. On the contrary, the strength of this study is its comprehensive nature of the surveillance, which helped to accurately determine the total number of tested patients (denominator) and quantify the changes before and after the beginning of the COVID-19 pandemic.

In conclusion, there was an apparent increase in the isolation rate defined in Japan and the frequency of infections defined in the WHO-GLASS for several bacterial species and specific AMRB, due to a substantial decrease in the denominator (i.e. the total number of tested patients). The results suggest that surveillance data during the COVID-19 pandemic should be interpreted carefully, not only by tracking changes in isolation rates, but also by considering both the numerator and denominator of the surveillance, especially the background factors that affect the denominator.

\section{Acknowledgements}

We are grateful to Ms. Emi Fujimura for her help in compiling the data. We are grateful to all the hospitals that participated and contributed data to JANIS as well as Editage (www.editage. jp) for English language editing.

\section{Author contributions}

Conceptualization: A.H., T.K., K.Y. Data curation and tabulation: K.Y. Funding acquisition: K.S., M. S. Investigation: A.H. Methodology: T.K., K.Y. Software: K.Y. Supervision: K.S., M.S. Validation: A.H., K.Y. Visualization: A.H. Writing - original draft: A. H.; writing - review \& editing: A.H., T.K., K.Y., K.S., M. S.

\section{Conflict of interest statement}

The authors have no conflicts of interest to disclose.

\section{Funding sources}

This study was supported by the Research Program on Emerging and Re-emerging Infectious Diseases from the Japan Agency for Medical Research and Development (AMED) under grant number JP21fk0108604.

\section{Appendix A. Supplementary data}

Supplementary data to this article can be found online at https://doi.org/10.1016/j.jhin.2021.09.011.

\section{References}

[1] Dona D, Di Chiara C, Sharland M. Multi-drug-resistant infections in the COVID-19 era: a framework for considering the potential impact. J Hosp Infect 2020;106(1):198-9.

[2] Rezasoltani S, Yadegar A, Hatami B, Asadzadeh Aghdaei H, Zali MR. Antimicrobial resistance as a hidden menace lurking behind the COVID-19 outbreak: the global impacts of too much hygiene on AMR. Front Microbiol 2020;11:590683.

[3] Monnet DL, Harbarth S. Will coronavirus disease (COVID-19) have an impact on antimicrobial resistance? Euro Surveill 2020; 25:2001886.

[4] Canton R, Gijon D, Ruiz-Garbajosa P. Antimicrobial resistance in ICUs: an update in the light of the COVID-19 pandemic. Curr Opin Crit Care 2020;26(5):433-41.

[5] Rodriguez-Alvarez M, Lopez-Vidal Y, Soto-Hernandez JL, MirandaNovales MG, Flores-Moreno K, Ponce de Leon-Rosales S. COVID-19: clouds over the antimicrobial resistance landscape. Arch Med Res 2021;52(1):123-6.

[6] Wee LEI, Conceicao EP, Tan JY, Magesparan KD, Amin IBM, Ismail BBS, et al. Unintended consequences of infection prevention and control measures during COVID-19 pandemic. Am J Infect Control 2021;49:469-77.

[7] Bentivegna E, Luciani M, Arcari L, Santino I, Simmaco M, Martelletti P. Reduction of multidrug-resistant (MDR) bacterial infections during the COVID-19 pandemic: a retrospective study. Int J Environ Res Public Health 2021;18. https: / / doi.org/10.3390/ ijerph18031003.

[8] Tiri B, Sensi E, Marsiliani V, Cantarini M, Priante G, Vernelli C, et al. Antimicrobial stewardship program, COVID-19, and infection control: spread of carbapenem-resistant Klebsiella Pneumoniae colonization in ICU COVID-19 patients. What did not work? J Clin Med 2020;9. https://doi.org/10.3390/jcm9092744.

[9] Kajihara T, Yahara K, Hirabayashi A, Shibayama K, Sugai M. current status, international collaboration, and future directions of a comprehensive antimicrobial resistance surveillance system. Jpn J Infect Dis 2021;74:87-96.

[10] Japan Nosocomial Infections Surveillance. Available at: https:// janismhlwgojp/report/kensahtml [last accessed March 2021].

[11] Hirabayashi A, Yahara K, Kajihara T, Sugai M, Shibayama K. Geographical distribution of Enterobacterales with a carbapenemase IMP-6 phenotype and its association with antimicrobial use: an analysis using comprehensive national surveillance data on antimicrobial resistance. PLoS One 2020;15(12):e0243630.

[12] Kajihara T, Yahara K, Stelling J, Eremin SR, Tornimbene B, Thamlikitkul V, et al. Comparison of de-duplication methods used by WHO Global Antimicrobial Resistance Surveillance System (GLASS) and Japan Nosocomial Infections Surveillance (JANIS) in 
the surveillance of antimicrobial resistance. PLoS One 2020; 15(6):e0228234.

[13] Global Antimicrobial Resistance Surveillance System (GLASS). Guide to preparing aggregated antimicrobial resistance data files. World Health Organization; 2016.

[14] Asia Pacific Observatory on Health Systems and Policies COVID-19 Health System Response Monitor. World Health organization. 2021.

[15] Marimuthu K, Pittet D, Harbarth S. The effect of improved hand hygiene on nosocomial MRSA control. Antimicrob Resist Infect Control 2014;3:34.

[16] Duckworth GJ, Jordens JZ. Adherence and survival properties of an epidemic methicillin-resistant strain of Staphylococcus aureus compared with those of methicillin-sensitive strains. J Med Microbiol 1990;32(3):195-200.

[17] McNeil JC, Flores AR, Kaplan SL, Hulten KG. The indirect impact of the SARS-CoV-2 pandemic on invasive group $A$ Streptococcus, Streptococcus Pneumoniae and Staphylococcus Aureus infections in Houston area children. Pediatr Infect Dis J 2021;40(8):e313-6.

[18] Rawson TM, Moore LSP, Zhu N, Ranganathan N, Skolimowska K, Gilchrist $M$, et al. Bacterial and fungal coinfection in individuals with coronavirus: a rapid review to support COVID-19 antimicrobial prescribing. Clin Infect Dis 2020;71(9):2459-68.

[19] Langford BJ, So M, Raybardhan S, Leung V, Westwood D, MacFadden DR, et al. Bacterial co-infection and secondary infection in patients with COVID-19: a living rapid review and meta-analysis. Clin Microbiol Infect 2020;26(12):1622-9.

[20] Duval A, Obadia T, Boelle PY, Fleury E, Herrmann JL, Guillemot D, et al. Close proximity interactions support transmission of ESBL-K. pneumoniae but not ESBL-E. coli in healthcare settings. PLoS Comput Biol 2019;15(5):e1006496.
[21] Harris AD, Kotetishvili M, Shurland S, Johnson JA, Morris JG, Nemoy LL, et al. How important is patient-to-patient transmission in extended-spectrum beta-lactamase Escherichia coli acquisition. Am J Infect Control 2007;35(2):97-101.

[22] Harris AD, Perencevich EN, Johnson JK, Paterson DL, Morris JG, Strauss SM, et al. Patient-to-patient transmission is important in extended-spectrum beta-lactamase-producing Klebsiella pneumoniae acquisition. Clin Infect Dis 2007;45(10):1347-50.

[23] Gurieva T, Dautzenberg MJD, Gniadkowski M, Derde LPG, Bonten MJM, Bootsma MCJ. The transmissibility of antibioticresistant Enterobacteriaceae in intensive care units. Clin Infect Dis 2018;66(4):489-93.

[24] Surgers L, Boyd A, Girard PM, Arlet G, Decre D. Biofilm formation by ESBL-producing strains of Escherichia coli and Klebsiella pneumoniae. Int J Med Microbiol 2019;309(1):13-8.

[25] Cornaglia G, Hryniewicz W, Jarlier V, Kahlmeter G, Mittermayer H, Stratchounski L, et al. European recommendations for antimicrobial resistance surveillance. Clin Microbiol Infect 2004;10(4):349-83.

[26] Rhee C, Klompas M. Sepsis trends: increasing incidence and decreasing mortality, or changing denominator? J Thorac Dis 2020;12(Suppl 1):S89-100.

[27] Rempel OR, Laupland KB. Surveillance for antimicrobial resistant organisms: potential sources and magnitude of bias. Epidemiol Infect 2009;137(12):1665-73.

[28] Janiec J, Evans MR, Thomas DR, Davies GH, Lewis H. Laboratorybased surveillance of Campylobacter and Salmonella infection and the importance of denominator data. Epidemiol Infect 2012;140(11):2045-52. 